\title{
A genetic risk score is differentially associated with migraine with and without aura
}

\author{
Claudia Pisanu $^{1,2} \cdot$ Martin Preisig $^{3} \cdot$ Enrique Castelao $^{3} \cdot$ Jennifer Glaus $^{4} \cdot$ \\ Giorgio Pistis $^{3}$ - Alessio Squassina ${ }^{2} \cdot$ Maria Del Zompo $^{2} \cdot$ Kathleen R. Merikangas $^{4}$. \\ Gérard Waeber $^{5} \cdot$ Peter Vollenweider $^{5} \cdot$ Jessica Mwinyi $^{1} \cdot$ Helgi B. Schiöth ${ }^{1}$
}

Received: 18 March 2017 / Accepted: 23 May 2017 / Published online: 27 June 2017

(c) The Author(s) 2017. This article is an open access publication

\begin{abstract}
Although a number of migraine-associated single-nucleotide polymorphisms (SNP) with small effect size have been identified, little is known about the additive impact of these variants on migraine risk, frequency and severity. We investigated to what extent a genetic risk score (GRS) based on recently published, novel migraineassociated SNPs is associated with migraine prevalence, subtypes and severity in a large population-based sample. The sample comprised 446 subjects with migraine and 2511 controls from the CoLaus/PsyCoLaus study. Fiftyfour SNPs earlier associated with migraine were selected. SNPs with a low impact on migraine prevalence in our sample were excluded using random forest. We combined the remaining 21 SNPs into a GRS and analyzed the
\end{abstract}

Jessica Mwinyi and Helgi B. Schiöth contributed equally to this work.

Electronic supplementary material The online version of this article (doi:10.1007/s00439-017-1816-5) contains supplementary material, which is available to authorized users.

Jessica Mwinyi

jessica.mwinyi@neuro.uu.se

1 Division of Functional Pharmacology, Department of Neuroscience, BMC, University of Uppsala, Husargatan 3, 75124 Uppsala, Sweden

2 Department of Biomedical Sciences, University of Cagliari, Cagliari, Italy

3 Department of Psychiatry, Lausanne University Hospital, Prilly, Switzerland

4 Genetic Epidemiology Research Branch, Intramural Research Program, National Institute of Mental Health, Bethesda, MD, USA

5 Department of Internal Medicine, Lausanne University Hospital, Lausanne, Switzerland association with migraine using logistic regression models. The GRS was significantly associated with migraine $(\mathrm{OR}=1.56, p=0.02)$ and migraine without aura (MWOA) $(\mathrm{OR}=2.01, p=0.003)$, but not with migraine with aura (MWA). The GRS was not associated with migraine frequency, intensity or interference with daily activities. We show that a GRS combining multiple genetic risk variants is associated with MWOA but not MWA, suggesting a different genetic susceptibility background underlying the two forms of migraine.

\section{Introduction}

Migraine is a disabling disorder characterized by recurrent episodes of headache attacks and associated symptoms. With a lifetime prevalence of $18 \%$ in women and $6 \%$ in men, migraine is an extremely common disorder (GBD 2015 Disease and Injury Incidence and Prevalence Collaborators 2016). Being ranked as the seventh most disabling disease in terms of years lived with disability, migraine has also a substantial socioeconomic impact (GBD 2015 Disease and Injury Incidence and Prevalence Collaborators 2016).

Migraine without aura (MWOA) is the most common form of migraine and is characterized by recurrent severe headache attacks lasting 4-72 h with associated gastrointestinal and autonomic symptoms [Headache Classification Committee of the International Headache Society (IHS) 2013; Headache Classification Subcommittee of the International Headache Society 2004]. Up to one-third of affected individuals experience transient focal neurological symptoms before or during the headache attack [(migraine with aura (MWA)] [Headache Classification Committee of the International Headache Society (IHS) 2013; Headache 
Classification Subcommittee of the International Headache 2004]. Although a number of hypotheses have been advanced, the pathogenesis of migraine is hitherto not fully understood, which is, however, of urgent need for the development of novel treatment approaches.

Migraine is thought to be the result of both genetic and environmental factors. The heritability of migraine has been estimated at 50\% (Mulder et al. 2003) and has been suggested to be different for the two subtypes. While firstdegree relatives of patients with MWOA show a 1.9 times higher risk for MWOA and a 1.4 times higher risk for MWA, relatives of individuals with MWA have a 4 times higher risk to develop MWA while the risk to develop MWOA is not elevated (Silberstein and Dodick 2013). In recent years, several single-nucleotide polymorphisms (SNP) significantly associated with migraine have been identified (Anttila et al. 2010, 2013; Chasman et al. 2011, 2014 Freilinger et al. 2012; Gormley et al. 2016). A recent meta-analysis pooling data from 29 genome-wide association studies (GWAS) identified 12 SNPs associated with increased migraine risk and, specifically, with larger effect sizes in MWOA compared to MWA cases (Anttila et al. 2013). However, other studies suggested an overlap between genetic variants associated with the two subtypes (Nyholt et al. 2015; Zhao et al. 2016).

The largest meta-analysis conducted so far on migraine genetics has just been recently published and included data of 59,674 migraine subjects and 316,078 controls. Besides the replication of some of the previously associated loci, this study identified 34 novel migraine-associated SNPs (Gormley et al. 2016). Notably, while this meta-analysis identified seven loci specifically associated with MWOA, a subset analysis revealed no significant association for MWA (Gormley et al. 2016). Therefore, the question to what extent a genetic heterogeneity exists between MWOA and MWA remains open. Importantly, all identified migraineassociated SNPs show small effect sizes and account for a small part of migraine heritability (Gormley et al. 2016). Thus, the application of genetic risk score (GRS) analysis, which sums risk alleles across multiple genetic loci, may be of essential help to more efficiently explain the missing heritability of complex phenotypes, as seen in migraine. Recently, a GRS study suggested distinct genetic signatures for migraine with and without comorbid major depressive disorder (MDD) (Ligthart et al. 2014). Other studies investigated the genetic overlap between migraine and ischemic stroke (Malik et al. 2015) or coronary artery disease (Winsvold et al. 2015), finding a much stronger overlap between cardiovascular diseases and MWOA rather than MWA.

Although first studies started to elucidate the isolated and combined effect of genetic variants on migraine risk, the aspect to what extent genetic susceptibility markers may predispose to a more severe form of migraine has only been scarcely investigated. To date, only one study tested the association between a cumulative GRS composed of seven SNPs and four proxies for severe migraine traits, i.e., early onset, $>100$ lifetime migraine attacks, prolonged migraine attacks and migraine chronification, in a clinical sample comprising 1806 migraine patients (Esserlind et al. 2016). Two SNPs, rs2274316 (MEF2D) and rs11172113 (LRP1), showed a significant association with high migraine attack frequency. However, the analysis evaluating the association between the GRS and "many lifetime attacks" or "prolonged migraine attacks" only showed a non-significant trend.

A cumulative genetic risk evaluation of migraine subtypes, i.e., MWA and MWOA, and additional important aspects of migraine severity, including associated disabling symptoms, migraine intensity, and interference with daily activities under consideration of very recently detected novel migraine-associated variants would add further understanding to the genotype-phenotype interplay in migraine. To close this gap of knowledge, we investigated the association between genetic susceptibility factors, taking recently described, novel genetic susceptibility markers into consideration, and migraine prevalence and severity in a large population-based cohort. In the current study, we aimed to specifically evaluate if (1) a GRS based on migraine-associated SNPs is associated with migraine and migraine-type prevalence in this large population-based sample and if (2) the GRS is associated with migraine frequency and distinct traits of migraine severity.

\section{Methods}

\section{Sample}

The present study included data from the CoLaus|PsyCoLaus study (Firmann et al. 2008; Preisig et al. 2009), a populationbased cohort study designed to investigate the genetic and environmental determinants of mental disorders and cardiovascular diseases (CVD) in the general population. Anthropometric measures, DNA and plasma samples were collected from a total of 6733 individuals (CoLaus), aged between 35 and 75 years, who were randomly selected between 2003 and 2006 according to the civil register of the city of Lausanne (Firmann et al. 2008). Sixty-seven percent of the participants of CoLaus in the age range of 35-66 years $(N=5535)$ also agreed to take part in the psychiatric evaluation (PsyCoLaus), resulting in a sample of 3719 individuals with both physical and psychiatric examination results (Preisig et al. 2009). The gender distribution of the PsyCoLaus sample $(47 \%$ men) was similar compared to that of the general population in the same age range (Preisig et al. 2009). Scores on the General Health Questionnaire (GHQ-12) (Goldberg 
1972), French translation (Bettschart and Bolognini 1996), a self-rating instrument completed at the somatic examination were similar for subjects who accepted to participate in PsyCoLaus and those who refused to participate. For the present analyses, we excluded 756 individuals because of missing genotyping data and 6 subjects because of missing lifetime MDD data, leaving a total of 2957 subjects. The CoLaus/PsyCoLaus was approved by the Institutional Ethics Committee of the University of Lausanne. Informed written consent was obtained from all participants.

\section{Assessment of clinical and biological data}

The lifetime prevalence of migraine was assessed according to the criteria of the International Classification of Headache Disorders (ICHD-II), using the validated French version of the Diagnostic Interview for Headache Syndromes (DIHS). For all the participants who reported headache symptoms in the validated French translation (Leboyer et al. 1995) of the Diagnostic Interview for Genetic Studies (DIGS) (Nurnberger et al. 1994), these symptoms were examined in detail using the DIHS interview. Subjects that satisfied the ICHD-II criteria were considered to be affected by migraine and were compared with subjects who did not meet the criteria o who did not report headache symptoms. The lifetime prevalence of MDD was assessed using the validated French translation (Leboyer et al. 1995) of the DIGS (Nurnberger et al. 1994). The French translation of the DIGS revealed excellent inter-rater reliability in terms of kappa and Yul's $Y$ coefficients for major mood and psychotic disorders and a slightly lower 6-week test-retest reliability (Preisig et al. 1999). Interviews were conducted by trained psychologists or psychiatrists and reviewed by an experienced senior psychologist. For each subject with a diagnosis of migraine, the following variables were extracted from the interview: age at onset, intensity of migraine (coded as a dichotomous variable comparing light/moderate versus severe migraine), migraine-associated gastrointestinal symptoms (loss of appetite, hunger, nausea, vomit, diarrhea and abdominal pain), duration of the interval between two attacks, duration of the attack, and interference with daily activities (evaluated with a score from 0 to 100$)$.

\section{Genotyping}

Nuclear DNA was extracted from whole blood of all participants included in the CoLaus study. Genome-wide genotyping was performed using Affymetrix 500K SNP chips. Subjects were excluded from the analysis in case of inconsistency between sex and genetic data, a genotype call rate less than $90 \%$, or inconsistencies of genotyping results in duplicate samples. Quality control for SNPs was performed using the following criteria: SNPs monomorphic among all samples; SNPs with call rates less than $95 \%$ deviation from the Hardy-Weinberg equilibrium (HWE) ( $p<1 \times 10 \mathrm{E}-07)$. Imputation was performed using minimac (http://genome.sph.umich.edu/wiki/Minimac) and 1000 Genomes (phase 1, version 3).

Fifty-four SNPs were chosen among previously genomewide determined, significant $(p<5 \times 10 \mathrm{E}-08)$ migraineassociated SNPs for which an odds ratio (OR) was reported by previous studies (Anttila et al. 2010, 2013; Freilinger et al. 2012; Gormley et al. 2016) (Supplementary Table 1). For 51 of these SNPs genotype data were available in our sample (Table 1). In case that an SNP was imputed, an imputation quality $\left(r^{2}-\right.$ hat $)>0.4$ was set as threshold for the inclusion of the variant into further analyses.

\section{Statistical analysis}

Differences in continuous or categorical variables between subjects with migraine and controls were assessed using Mann-Whitney test or Pearson's Chi-square test, respectively.

Table 1 Demographic and clinical characteristics of the sample $(n=2957)$

\begin{tabular}{|c|c|c|c|c|c|c|c|c|c|c|}
\hline & \multicolumn{5}{|c|}{ Migraine vs. controls } & \multicolumn{3}{|c|}{ MWA vs. controls } & \multicolumn{2}{|c|}{$\begin{array}{l}\text { MWOA vs. con- } \\
\text { trols }\end{array}$} \\
\hline & Controls & Migraine & Statistics & $p$ & MWA & Statistics & $p$ & MWOA & Statistics & $p$ \\
\hline$N$ & 2511 & 446 & - & - & 152 & & - & 294 & & - \\
\hline Age (years) & $51.7( \pm 8.9)$ & $50.6( \pm 8.6)$ & 519,856 & 0.016 & $51.1( \pm 9.0)$ & 183,737 & 0.44 & $48.4( \pm 8.3)$ & 336,119 & 0.012 \\
\hline Sex female $(\%)$ & $50 \%$ & $69 \%$ & 55.27 & $<0.001$ & $60 \%$ & 5.34 & 0.02 & $74 \%$ & 60.47 & $<0.001$ \\
\hline Lifetime MDD (\%) & $41 \%$ & $57 \%$ & 41.47 & $<0.001$ & $63 \%$ & 29.45 & $<0.001$ & $54 \%$ & 19.09 & $<0.001$ \\
\hline
\end{tabular}

Continuous variables are expressed as mean \pm standard deviation; $p$ values are calculated using Mann-Whitney test or Pearson's Chi-square test, comparing subjects with migraine or migraine subtypes vs. controls

Significant differences are indicated in bold

$M D D$ major depressive disorder, $M W A$ migraine with aura, $M W O A$ migraine without aura 
Linkage disequilibrium (LD) between markers was measured by the r-squared coefficient using Haploview (v. 4.2) (Barrett et al. 2005). SNPs with $r^{2}$ values larger than 0.8 were excluded. Deviation from HWE was tested using Chi-square test $(p<0.001)$. HWE $p$ value threshold was corrected according to Bonferroni $(p<0.001$, i.e., $0.05 / 51$ tested genetic variants). SNPs with a minor allele frequency $(\mathrm{MAF})<0.01$ were also excluded.

Genotypes for 51 out of 54 SNPs previously associated with migraine and for which an OR had been reported were available in our dataset (Supplementary Table 1). Two SNPs were not in HWE and were excluded. After the removal of SNPs with too low imputation quality $(n=0)$, with MAF $<0.01(n=0)$ and of SNPs being in LD $(n=9)$, we proceeded with 40 SNPs in subsequent analyses.

The association between individual SNPs and migraine was tested using logistic regression analyses adjusting for age, sex and a lifetime diagnosis of MDD. A Bonferroniadjusted $p$ value of $0.0013(0.05 / 40)$ was applied.

Analyses were performed applying an additive genetic model. The genotype of each SNP was coded based on the amount of effect alleles, i.e., 0 for no effect alleles, 1 for heterozygous SNP carriers and 2 for individuals carrying two effect alleles. Subsequently, SNP-associated beta coefficients were calculated based on the ORs reported in Anttila et al. 2013, Freilinger et al. 2012 and Gormley et al. 2016, and multiplied with the amount of alleles to obtain weighted SNP scores. A random forest model was constructed including weighted scores as predictors and a lifetime diagnosis of migraine as the target variable. The dataset was randomly partitioned to a training (70\%), validation $(15 \%)$ and testing $(15 \%)$ dataset to obtain a better unbiased estimate of error. A total of 10,000 random decision trees were created. SNPs with a positive mean decrease accuracy (i.e., variables that cause subjects to be incorrectly classified if removed) were considered to have a relevant influence on the model and were chosen for inclusion in the GRS.

GRS was calculated by summing up the weighted SNP scores according to the following formula:

$\sum_{i=1}^{n} k$ effect alleles $\mathrm{SNP} i \times$ beta value $_{\mathrm{SNP} i}$

with $n$ the number of included SNPs in the model, and $k$ the number of effect alleles. The load of effect alleles between subjects with migraine or migraine subtypes and controls was compared using the Mann-Whitney test.

The GRS was used as a continuous predictor in a binary logistic regression model with migraine (or migraine subtypes) as the dependent variable, adjusting for age, sex, and a lifetime diagnosis of MDD. The GRS was also coded as a dichotomous variable to compare the association between migraine and the upper/lower quartiles of the GRS distribution using a binary logistic regression model.

In a second step, we ran a case-only analysis to test the association between the GRS and characteristics of migraine severity. Association between GRS and age at onset, migraine frequency, duration of attacks, or interference with daily activities was tested performing Spearman's correlation analyses, while the association between GRS and migraine intensity or gastrointestinal symptoms was tested with the Mann-Whitney test. To test the association between the GRS and continuous or dichotomous clinical characteristics of migraine, we constructed linear or logistic regression models, respectively, adjusting for age, sex and lifetime MDD. A $p$ value $<0.05$ was considered significant. Analyses were performed using Haploview, the package Rattle (Graham 2011) in R (v. 3.2.3), SPSS v. 21 (IBM, Armonk, NY, USA), and GraphPad Prism 5.

\section{Results}

\section{Demographic and clinical characteristics of the sample}

The demographic and clinical characteristics of the sample are shown in Table 1. Within the sample of 2957 participants, 446 subjects (15\%) met the lifetime diagnostic criteria for migraine. Among them, 34\% fulfilled the criteria for MWA. Subjects with migraine were more likely to be younger, female, and to have a higher lifetime prevalence of MDD (Table 1). Therefore, analyses were adjusted for these variables.

\section{Isolated SNPs have only a small impact on migraine risk}

We first tested the association between migraine and the 40 genetic variants individually in unadjusted analyses applying an additive genetic model and using logistic regression analysis (Supplementary Table 2).

Unadjusted analyses showed that two SNPs were associated with migraine at a Bonferroni corrected threshold of $0.0013(0.05 / 40)$ : rs 4814864 , located in the SLC24A3 gene $(\mathrm{OR}=1.32, p=0.0008)$, and $\mathrm{rs} 186166891$, located in the $S U G C T$ gene $(\mathrm{OR}=1.48, p=0.0004)$. One genetic variant was associated with migraine at a nominal level $(\mathrm{rs} 1024905, \mathrm{OR}=1.19, p=0.016)$.

Subsequently, we applied a logistic regression analysis with migraine as the outcome variable, adjusting for age, sex, and a lifetime diagnosis of MDD. The association of rs186166891 with migraine was confirmed $(\mathrm{OR}=1.54$, $p=0.0002)$, while rs4814864 was associated with migraine only at a nominal level $(\mathrm{OR}=1.30, p=0.0017)$. 
The SNP rs186166891 was associated with MWOA (unadjusted OR $=1.58, p=0.0004$; adjusted OR: 1.66, $p=0.0001$ ), but not with MWA (adjusted OR: 1.32, $p=0.15)$.

Analyses conducted in migraine subtypes also showed an association between rs1024905 and MWOA (unadjusted $\mathrm{OR}=1.41, p=0.0001 ;$ adjusted $\mathrm{OR}=1.42, p=0.0001)$, and a nominal association between rs4814864 and MWOA (unadjusted OR $=1.27, p=0.017$ ). Only one SNP was nominally associated with MWA (rs4814864, unadjusted $\mathrm{OR}=1.41, p=0.008$; adjusted $\mathrm{OR}=1.37, p=0.015)$. None of the other variants was associated with migraine or migraine subtypes. These results pointed to a relatively small impact of single SNPs on migraine and migraine subtypes in our population-based cohort.

\section{A polygenic risk score composed of susceptibility SNPs for migraine is significantly associated with MWOA}

To weight the genetic variants with regard to their impact on migraine outcome, a random forest algorithm was developed. As shown in Table 2 and Fig. 1, a total of 21 genetic variants (closest genes: MEF2D, PRDM16, TSPAN2,
TRPM8, CARF, TGFBR2, PHACTR1, NOTCH4, SUGCT, DOCK4, PLCE1, HPSE2, ARMS2, MRVI1, LRP1, ITPK1, ZCCHC14, NLRP1, and SLC24A3) induced a positive mean decrease accuracy and were considered in the subsequent calculation of the weighted GRS. The study participants showed a GRS distribution ranging from -1.33 to 0.76 .

Mann-Whitney test showed that subjects with migraine or MWOA, but not MWA, had a higher load of risk alleles compared to controls (Fig. 2). As shown in Table 3, binary logistic regression analysis confirmed the significant impact of the GRS on the diagnosis of migraine. The covariates female sex, younger age, and a lifetime diagnosis of MDD were also significantly associated with a diagnosis of migraine.

Importantly, when stratifying migraine according to the main clinical subtypes MWA and MWOA, only MWOA appeared to be significantly impacted by the GRS (Table 3). When the GRS was dichotomized to compare migraine prevalence between the bottom and top quartiles of the GRS distribution, the GRS was still associated with migraine [OR: 1.45 (95\% confidence interval $=1.09-1.93), p=0.011]$ and MWOA [OR: $1.76(95 \%$

Table 2 SNPs previously associated with migraine and included in the GRS

\begin{tabular}{|c|c|c|c|c|c|c|c|c|}
\hline SNP & $\mathrm{Chr}$ & Location & Gene & MA & MAF & Reported OR (95\% CI) & Beta coefficient ${ }^{\mathrm{b}}$ & References \\
\hline rs2274316 & 1 & Genic & $M E F 2 D$ & $\mathrm{C}$ & 0.37 & $1.07(1.04-1.09)$ & 0.07 & Anttila et al. 2013 \\
\hline rs 10218452 & 1 & Genic & PRDM16 & G & 0.22 & $1.11(1.10-1.13)$ & 0.10 & Gormley et al. 2016 \\
\hline rs2078371 & 1 & Intergenic & Near TSPAN2 & $\mathrm{C}$ & 0.12 & $1.11(1.09-1.13)$ & 0.10 & Gormley et al. 2016 \\
\hline rs7577262 & 2 & Genic & TRPM 8 & A & 0.10 & $0.87(0.84-0.90)$ & -0.14 & Anttila et al. 2013 \\
\hline rs 17862920 & 2 & Genic & TRPM8 & $\mathrm{T}$ & 0.10 & $0.77(0.70-0.84)$ & -0.26 & Freilinger et al. 2012 \\
\hline rs 10166942 & 2 & Intergenic & Near TRPM8 & $\mathrm{C}$ & 0.20 & $0.94(0.89-0.99)$ & -0.06 & Gormley et al. 2016 \\
\hline rs138556413 & 2 & Genic & $C A R F$ & $\mathrm{~T}$ & 0.03 & $0.88(0.84-0.92)$ & -0.13 & Gormley et al. 2016 \\
\hline rs6790925 & 3 & Intergenic & Near $T G F B R 2$ & $\mathrm{~T}$ & 0.38 & $1.15(1.10-1.21)$ & 0.14 & Anttila et al. 2013 \\
\hline rs9349379 & 6 & Genic & PHACTR1 & G & 0.41 & $0.93(0.92-0.95)$ & -0.07 & Gormley et al. 2016 \\
\hline rs9267918 & 6 & Intergenic & Near NOTCH4 & A & 0.06 & $0.91(0.88-0.94)$ & -0.09 & Gormley et al. 2016 \\
\hline rs186166891 & 7 & Genic & $S U G C T$ & $\mathrm{~T}$ & 0.11 & $1.09(1.07-1.12)$ & 0.09 & Gormley et al. 2016 \\
\hline rs 10155855 & 7 & Intergenic & Near DOCK4 & $\mathrm{T}$ & 0.05 & $1.08(1.05-1.12)$ & 0.08 & Gormley et al. 2016 \\
\hline rs 10786156 & 10 & Genic & $P L C E 1$ & G & 0.45 & $0.95(0.94-0.96)$ & -0.05 & Gormley et al. 2016 \\
\hline rs 12260159 & 10 & Genic & HPSE2 & A & 0.07 & $0.92(0.89-0.94)$ & -0.08 & Gormley et al. 2016 \\
\hline rs2223089 & 10 & Intergenic & Near ARMS2 & $\mathrm{C}$ & 0.08 & $0.93(0.91-0.95)$ & -0.07 & Gormley et al., 2016 \\
\hline rs4910165 & 11 & Genic & MRVII & $\mathrm{C}$ & 0.33 & $0.94(0.91-0.98)$ & -0.06 & Gormley et al. 2016 \\
\hline rs11172113 & 12 & Genic & $L R P 1$ & $\mathrm{C}$ & 0.42 & $0.90(0.89-0.91)$ & -0.11 & Gormley et al. 2016 \\
\hline rs11624776 & 14 & Intergenic & Near ITPKI & $\mathrm{C}$ & 0.31 & $0.96(0.94-0.97)$ & -0.04 & Gormley et al. 2016 \\
\hline rs4081947 & 16 & Intergenic & Near ZCCHC14 & G & 0.34 & $1.03(1.00-1.06)$ & 0.03 & Gormley et al. 2016 \\
\hline rs 75213074 & 17 & Intergenic & Near NLRP1 & $\mathrm{T}$ & 0.03 & $0.89(0.86-0.93)$ & -0.12 & Gormley et al. 2016 \\
\hline rs4814864 & 20 & Genic & $S L C 24 A 3$ & $\mathrm{C}$ & 0.26 & 1.07 (1.06-1.09) & 0.07 & Gormley et al. 2016 \\
\hline
\end{tabular}

${ }^{\text {a }}$ In the meta-analysis by Gormley et al. (2016), the association is reported for rs140002913 (now merged into rs9267918)

b The beta coefficients column shows natural logarithms of the ORs reported by previous studies

$C h r$ chromosome, $C I$ confidence interval, $M A$ minor allele, $M A F$ minor allele frequency, $O R$ odds ratio, $S N P$ single-nucleotide polymorphism 
Fig. 1 Random forest model including 21 SNPs previously associated with migraine. The first 30 variables with the highest mean decrease accuracy are plotted. Twenty-one migraineassociated SNP were shown to induce a positive change in mean decrease accuracy. These SNPs were thus considered to have a relevant influence on the model and were chosen for inclusion in the GRS. GRS genetic risk score, SNP singlenucleotide polymorphism
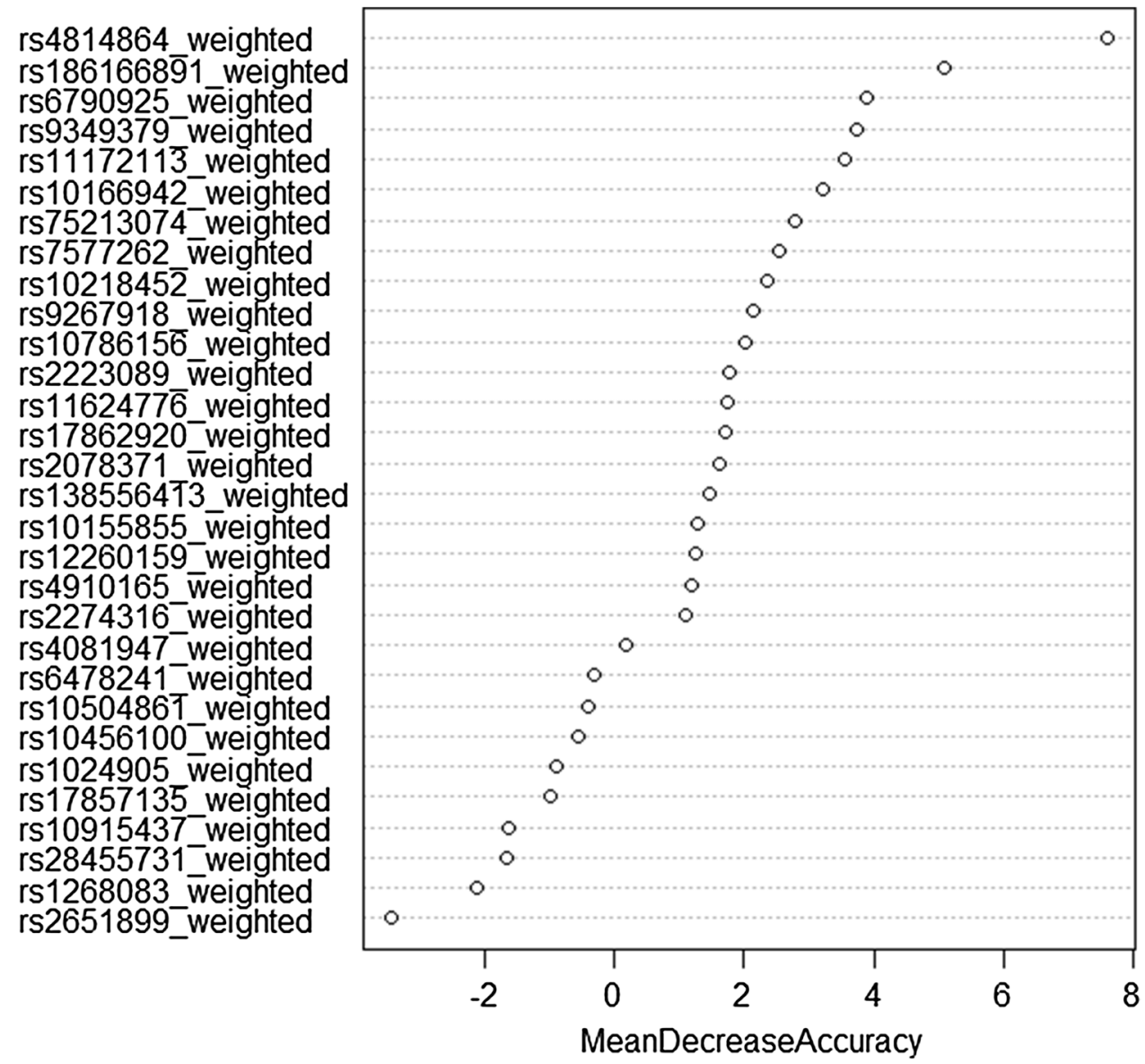

impacts the prevalence of migraine subtypes and migraine severity in a large population-based sample, adjusting for demographic and clinical confounders. We demonstrate that the GRS based on SNPs previously identified in GWAS studies or meta-analyses to be associated with migraine specifically impacts MWOA, but not the risk for MWA. These findings allow the suggestion that MWOA and MWA have a different genetic susceptibility background that plays a role in their pathogenesis.

First-degree relatives of individuals with MWA show a considerably higher risk of MWA (4 times) compared to the higher risk of MWOA shown by first-degree relatives of MWOA patients (1.9 times) (Silberstein and Dodick 2013). On the basis of these estimates, the genetic contribution to migraine pathogenesis has been hypothesized to be higher for MWA compared to MWOA. However, a recent metaanalysis of GWAS, including data from 23,285 migraine cases and 95,425 controls, failed to identify genetic variants significantly predisposing to MWA (Anttila et al. 2013). Since this meta-analysis only evaluated the association of common SNPs, a possible explanation for this inconsistency lays in the fact that genetic predisposition to MWA could be mediated by rare variants with large effect size (Anttila et al. 2013). A recent reanalysis of the same data 


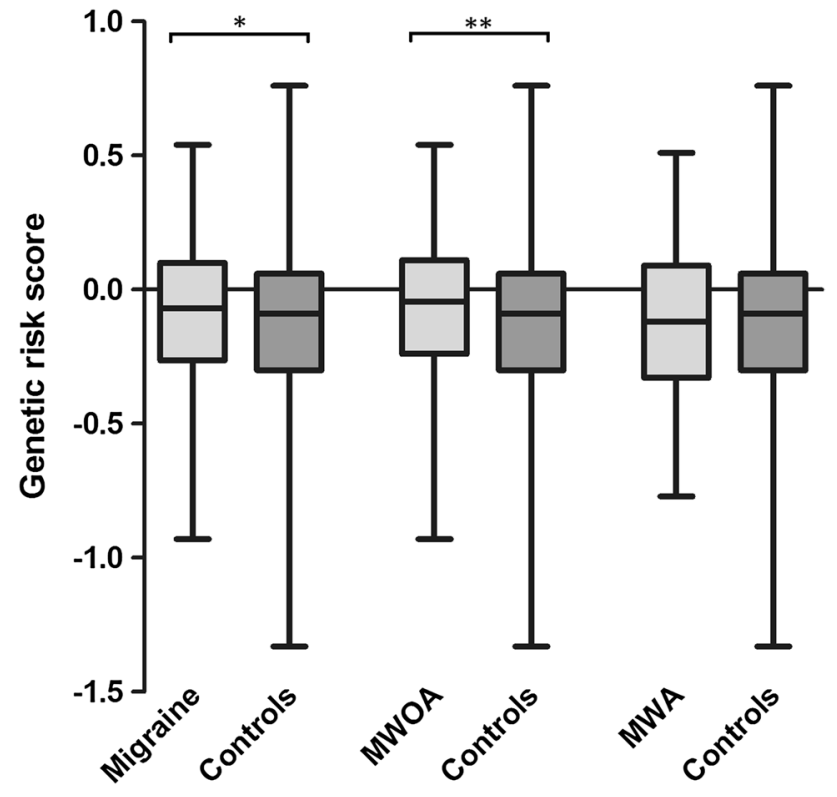

Fig. 2 Box and whisker plot of GRS in subjects with migraine or migraine subtypes compared to controls. Mann-Whitney test showed a significant association between the genetic risk score and migraine ( $U=522,400, p=0.02)$ or MWOA $(U=329,900, p=0.003)$, but not the MWA subtype $(U=189,200, p=0.8)$. $*<0.05$, **< $<0.005$. GRS genetic risk score, MWA migraine with aura, MWOA migraine without aura

found significant heterogeneity of SNP effects between MWA and MWOA, although the direction of effect for the majority of selected SNPs was found to be the same between the two subtypes (Nyholt et al. 2015). The largest meta-analysis conducted so far, including data from 59,674 affected subjects and 316,078 controls, also failed to identify SNPs associated with MWA (Gormley et al. 2016). Our results support the hypothesis that common variants with small effect sizes, even when evaluating them additively by GRS analysis, do not show an impact on MWA diagnosis.

Among the new susceptibility loci identified by Gormley et al. (Gormley et al. 2016) and included in our GRS, two lie in or near genes involved in regulation of vascular tone (MEF2D, DOCK4, and SLC24A3) (Dong et al. 2006; Firulli et al. 1996; Kang et al. 2015), while $A R M S 2$, PHACTR1, TGFBR2, LRP1, and NOTCH4 have been previously associated with vascular disease (Delev et al. 2017; Beaudoin et al. 2015; Guo et al. 2016; Hayashi et al. 2010; Yang et al. 2016), consistently with the suggested role of vascular dysfunction in migraine pathogenesis. Interestingly, one of the novel migraine-associated SNPs included in our GRS, rs75213074, is located near NLRP1, which encodes for the sensor component of an inflammasome (a protein complex that activates caspases and cytokines in response to damage-associated signals) (Guo et al. 2015). As outlined by a recent review, due to their ability to integrate various inflammatory signals and their expression in endothelial cells, neurons, and astrocytes, inflammasomes represent a promising functional link between vascular and neurological diseases (Lenart et al. 2016).

Similarly to other complex phenotypes, migraine heritability appears to be due to a large number of weak associations with many genetic variants, rather than few common risk variants (Anttila et al. 2013; Chasman et al. 2016). Therefore, a GRS evaluating the cumulative effect of multiple variants with a small impact is more likely to be able to explain a part of migraine missing heritability.

To date, only a few studies evaluated the association between a GRS and migraine prevalence in a populationbased sample. Rodriguez-Acevedo and coworkers found an association between migraine and a GRS (including 51 genetic variants previously nominally associated with migraine) in a small sample including 74 cases and 211 controls (Rodriguez-Acevedo et al. 2015). We used a different approach by selecting only genetic variants previously associated with migraine with a genome-wide significant threshold for which an OR had been reported and we further prioritized variables using a random forest algorithm. Another study investigated the overlap between migraine and MDD, showing that the subgroup of patients with migraine and comorbid MDD are genetically most
Table 3 Association between GRS and diagnosis of migraine and migraine subtypes according to logistic regression models

\begin{tabular}{|c|c|c|c|c|c|c|}
\hline & \multirow{2}{*}{\multicolumn{2}{|c|}{$\frac{\text { Migraine }(N=446)}{\text { vs. controls }(2511)}$}} & \multicolumn{2}{|l|}{ MWA $(N=152)$} & \multicolumn{2}{|l|}{ MWOA $(N=294)$} \\
\hline & & & & & & \\
\hline & OR $(95 \% \mathrm{CI})$ & $p$ & OR $(95 \% \mathrm{CI})$ & $p$ & OR $(95 \% \mathrm{CI})$ & $p$ \\
\hline GRS & $1.56(1.07-2.27)$ & 0.020 & $0.96(0.54-1.72)$ & 0.890 & $2.01(1.28-3.17)$ & 0.003 \\
\hline Female sex & $2.04(1.63-2.55)$ & $<0.001$ & $1.22(0.86-1.72)$ & 0.260 & $2.73(2.06-3.61)$ & $<0.001$ \\
\hline Age & $0.99(0.97-1.00)$ & 0.010 & $1.00(0.98-1.01)$ & 0.590 & $0.98(0.97-0.99)$ & 0.004 \\
\hline MDD & $1.63(1.32-2.01)$ & $<0.001$ & $2.37(1.67-3.36)$ & $<0.001$ & $1.34(1.04-1.72)$ & 0.023 \\
\hline
\end{tabular}

Significant differences are indicated in bold

$C I$ confidence interval, GRS genetic risk score, $M D D$ major depressive disorder, $M W A$ migraine with aura, $M W O A$ migraine without aura, $O R$ odds ratio 


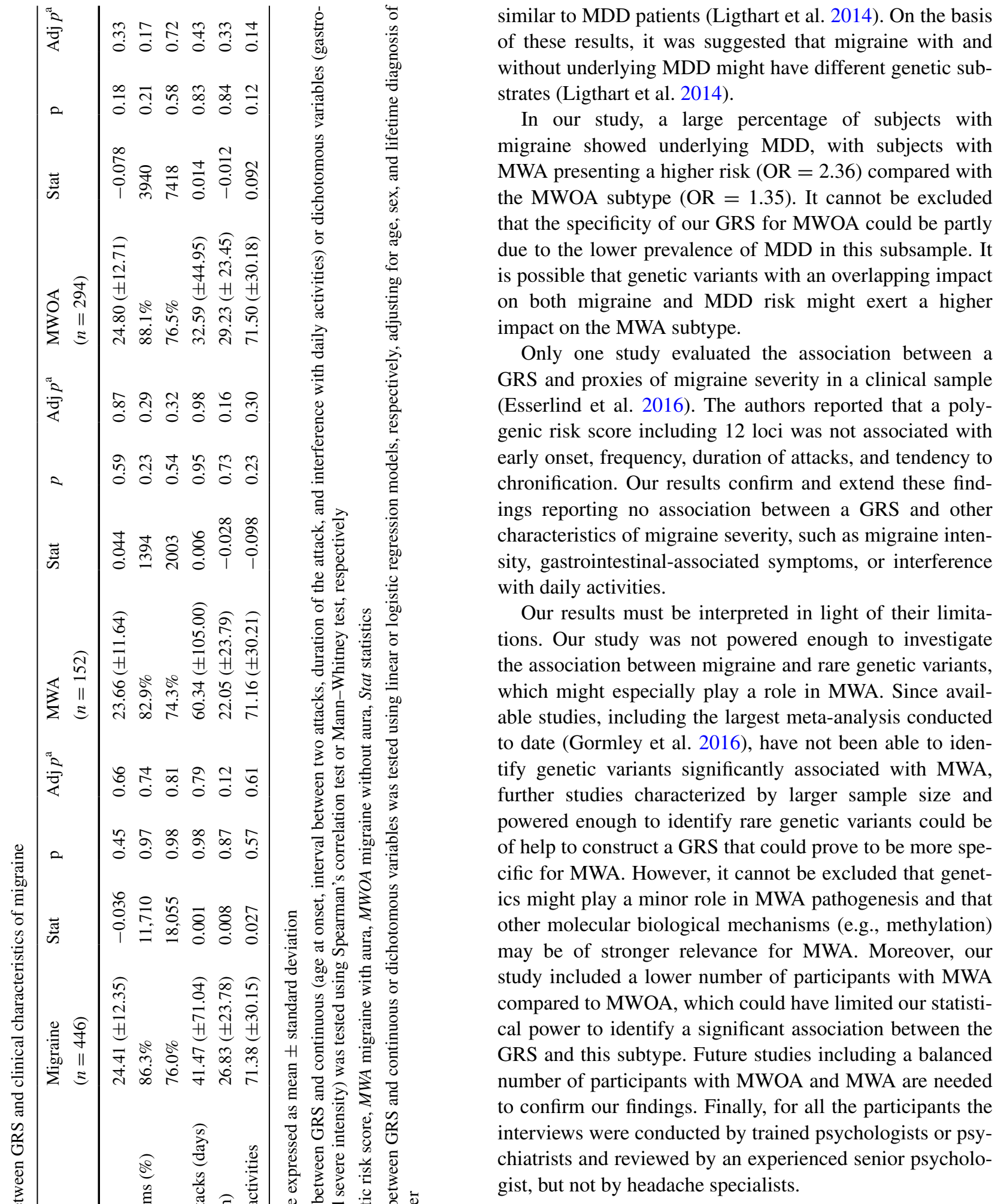

similar to MDD patients (Ligthart et al. 2014). On the basis of these results, it was suggested that migraine with and without underlying MDD might have different genetic subtrates (Ligthart et al. 2014).

In our study, a large percentage of subjects with migraine showed underlying MDD, with subjects with MWA presenting a higher risk $(\mathrm{OR}=2.36)$ compared with the MWOA subtype $(\mathrm{OR}=1.35)$. It cannot be excluded hat the specificity of our GRS for MWOA could be partly due to the lower prevalence of MDD in this subsample. It is possible that genetic variants with an overlapping impact on both migraine and MDD risk might exert a higher mpact on the MWA subtype.

Only one study evaluated the association between a GRS and proxies of migraine severity in a clinical sample Esserlind et al. 2016). The authors reported that a polyearly onset, frequency, duration of attacks, and tendency to chronification. Our results confirm and extend these findings reporting no association between a GRS and other ith daily activities.

Our results must be interpreted in light of their limitatons. Our study was not powered enough to investigate which might especially play a role in MWA. Since available studies, including the largest meta-analysis conducted date (Gormley et al. 2016), have not been able to idenify genetic variants significantly associated with MWA, urther studies characterized by larger sample size and powered enough to identify rare genetic variants could be of help to construct a GRS that could prove to be more speific for MWA. However, it cannot be excluded that genetics might play a minor role in MWA pathogenesis and that other molecular biological mechanisms (e.g., methylation) may be of stronger relevance for MWA. Moreover, our study included a lower number of participants with MWA compared to MWOA, which could have limited our statistical power to identify a significant association between the GRS and this subtype. Future studies including a balanced number of participants with MWOA and MWA are needed to confirm our findings. Finally, for all the participants the nterviews were conducted by trained psychologists or psygist, but not by headache specialists.

\section{Conclusion}

In conclusion, the present study suggests that a GRS combining the effect of multiple loci is associated with MWOA 
but not MWA in a large population-based sample. Genetic risk variants so far detected to play a role in migraine are not able to explain a comprehensive set of clinical characteristics of migraine severity.

\section{Compliance with ethical standards}

Funding This study was supported by a grant from the Swedish Society for Medical Research (SSMF) to JM, a grant from the Swedish Research Council to HBS, and in part by the Intramural Research Program of the National Institute of Mental Health (ZIAMH002932). The CoLaus/PsyCoLaus study was and is supported by research grants from GlaxoSmithKline, the Faculty of Biology and Medicine of Lausanne, the Swiss National Science Foundation (Grants 3200B0-105993, 3200B0-118308, 33CSCO-122661, 33CS30-139468, and 33CS30148401).

Conflict of interest The authors declare that they have no conflict of interest.

Ethical approval All procedures performed in studies involving human participants were in accordance with the ethical standards of the institutional and/or national research committee and with the 1964 Helsinki Declaration and its later amendments or comparable ethical standards.

Informed consent Informed consent was obtained from all individual participants included in the study.

Open Access This article is distributed under the terms of the Creative Commons Attribution 4.0 International License (http://creativecommons.org/licenses/by/4.0/), which permits unrestricted use, distribution, and reproduction in any medium, provided you give appropriate credit to the original author(s) and the source, provide a link to the Creative Commons license, and indicate if changes were made.

\section{References}

Anttila V et al (2010) Genome-wide association study of migraine implicates a common susceptibility variant on $8 \mathrm{q} 22.1$. Nat Genet 42:869-873. doi:10.1038/ng.652

Anttila V et al (2013) Genome-wide meta-analysis identifies new susceptibility loci for migraine. Nat Genet 45:912-917. doi:10.1038/ng.2676

Barrett JC, Fry B, Maller J, Daly MJ (2005) Haploview: analysis and visualization of LD and haplotype maps. Bioinformatics 21:263265. doi:10.1093/bioinformatics/bth457

Beaudoin $M$ et al (2015) Myocardial infarction-associated SNP at 6p24 interferes with MEF2 binding and associates with PHACTR1 expression levels in human coronary arteries. Arterioscler Thromb Vasc Biol 35:1472-1479. doi:10.1161/ ATVBAHA.115.305534

Bettschart W, Bolognini M (1996) Questionnaire de santé GHQ-12. Médicales Pierre Fabre, Boulogne

Chasman DI et al (2011) Genome-wide association study reveals three susceptibility loci for common migraine in the general population. Nat Genet 43:695-698. doi:10.1038/ng.856
Chasman DI, Anttila V, Buring JE, Ridker PM, Schurks M, Kurth $\mathrm{T}$, International Headache Genetics C (2014) Selectivity in genetic association with sub-classified migraine in women. PLoS Genet 10:e1004366. doi:10.1371/journal.pgen.1004366

Chasman DI, Schurks M, Kurth T (2016) Population-based approaches to genetics of migraine. Cephalalgia Int J Headache 36:692-703. doi:10.1177/0333102416638519

Delev D et al (2017) NOTCH4 gene polymorphisms as potential risk factors for brain arteriovenous malformation development and hemorrhagic presentation. J Neurosurg 126:1552-1559. doi:10.3171/2016.3.JNS151731

Dong H, Jiang Y, Triggle CR, Li X, Lytton J (2006) Novel role for $\mathrm{K}+$-dependent $\mathrm{Na}+/ \mathrm{Ca} 2+$ exchangers in regulation of cytoplasmic free $\mathrm{Ca} 2+$ and contractility in arterial smooth muscle. Am J Physiol Heart Circ Physiol 291:H1226-H1235. doi:10.1152/ajpheart.00196.2006

Esserlind AL et al (2016) The association between candidate migraine susceptibility loci and severe migraine phenotype in a clinical sample. Cephalalgia Int J Headache 36:615-623. doi: $10.1177 / 0333102415570492$

Firmann M et al (2008) The CoLaus study: a population-based study to investigate the epidemiology and genetic determinants of cardiovascular risk factors and metabolic syndrome. BMC Cardiovasc Disord 8:6. doi:10.1186/1471-2261-8-6

Firulli AB, Miano JM, Bi W, Johnson AD, Casscells W, Olson EN, Schwarz JJ (1996) Myocyte enhancer binding factor-2 expression and activity in vascular smooth muscle cells. Association with the activated phenotype. Circ Res 78:196-204

Freilinger T et al (2012) Genome-wide association analysis identifies susceptibility loci for migraine without aura. Nat Genet 44:777-782. doi:10.1038/ng.2307

GBD 2015 Disease and Injury Incidence and Prevalence Collaborators (2016) Global, regional, and national incidence, prevalence, and years lived with disability for 310 diseases and injuries, 1990 2015: a systematic analysis for the global burden of disease study 2015. Lancet 388:1545-1602. doi:10.1016/S0140-6736

Goldberg D (1972) The detection of psychiatric illness by questionnaire: A technique for the identification and assessment of nonpsychotic psychiatric illness. Oxford University Press, London

Gormley P et al (2016) Meta-analysis of 375,000 individuals identifies 38 susceptibility loci for migraine. Nat Genet 48:856-866. doi:10.1038/ng.3598

Graham W (2011) Data mining with rattle and R: the art of excavating data for knowledge discovery. Springer, Berlin

Guo H, Callaway JB, Ting JP (2015) Inflammasomes: mechanism of action, role in disease, and therapeutics. Nat Med 21:677687. doi: $10.1038 / \mathrm{nm} .3893$

Guo DC et al (2016) Genetic variants in LRP1 and ULK4 are associated with acute aortic dissections. Am J Hum Genet 99:762769. doi:10.1016/j.ajhg.2016.06.034

Hayashi $\mathrm{H}$ et al (2010) $\mathrm{CFH}$ and ARMS2 variations in age-related macular degeneration, polypoidal choroidal vasculopathy, and retinal angiomatous proliferation. Investig Ophthalmol Vis Sci 51:5914-5919. doi:10.1167/iovs.10-5554

Headache Classification Committee of the International Headache Society (IHS) (2013) The international classification of headache disorders, 3rd edition (beta version). Cephalalgia Int $\mathbf{J}$ Headache 32:629-808. doi:10.1177/0333102413485658

Headache Classification Subcommittee of the International Headache Society (2004) The international classification of headache disorders, 2nd edn. Cephalalgia Int J Headache 24(Suppl 1):9-160

Kang JH et al (2015) The efficacy of low-dose transdermal fentanyl in opioid-naive cancer patients with moderate-to-severe pain. Korean J Intern Med 30:88-95. doi:10.3904/kjim.2015.30.1.88 
Leboyer MBB, Gorwood P, Teherani M, Allilaire JF, Preisig M, Matthey ML, Poyetton V, Ferrero F (1995) Interview Diagnostique Pour Les Études Génétiques. INSERM, Paris

Lenart N, Brough D, Denes A (2016) Inflammasomes link vascular disease with neuroinflammation and brain disorders. J Cereb Blood Flow Metab Off J Int Soc Cereb Blood Flow Metab. doi:10.1177/0271678X16662043

Ligthart L et al (2014) Genetic risk score analysis indicates migraine with and without comorbid depression are genetically different disorders. Hum Genet 133:173-186. doi:10.1007/ s00439-013-1370-8

Malik R et al (2015) Shared genetic basis for migraine and ischemic stroke: a genome-wide analysis of common variants. Neurology 84:2132-2145. doi:10.1212/WNL.0000000000001606

Mulder EJ et al (2003) Genetic and environmental influences on migraine: a twin study across six countries. Twin Res Off J Int Soc Twin Stud 6:422-431. doi:10.1375/136905203770326420

Nurnberger JI Jr. et al (1994) Diagnostic interview for genetic studies. Rationale, unique features, and training. NIMH Genet Initiat Arch Gen Psychiatry 51:849-859 (discussion 863-844)

Nyholt DR et al (2015) Concordance of genetic risk across migraine subgroups: impact on current and future genetic association studies. Cephalalgia Int J Headache 35:489-499. doi: $10.1177 / 0333102414547784$
Preisig M, Fenton BT, Matthey ML, Berney A, Ferrero F (1999) Diagnostic interview for genetic studies (DIGS): inter-rater and test-retest reliability of the French version. Eur Arch Psychiatry Clin Neurosci 249:174-179

Preisig M et al (2009) The PsyCoLaus study: methodology and characteristics of the sample of a population-based survey on psychiatric disorders and their association with genetic and cardiovascular risk factors. BMC Psychiatry 9:9. doi:10.1186/1471-244X-9-9

Rodriguez-Acevedo AJ et al (2015) Common polygenic variation contributes to risk of migraine in the Norfolk Island population. Hum Genet 134:1079-1087. doi:10.1007/s00439-015-1587-9

Silberstein SD, Dodick DW (2013) Migraine genetics: part II. Headache 53:1218-1229. doi:10.1111/head.12169

Winsvold BS et al (2015) Genetic analysis for a shared biological basis between migraine and coronary artery disease. Neurol Genet 1:e10. doi:10.1212/NXG.0000000000000010

Yang $M$ et al (2016) Polymorphisms of TGFbeta-1 and TGFBR2 in relation to coronary artery disease in a Chinese population. Clin Biochem 49:873-878. doi:10.1016/j.clinbiochem.2016.05.022

Zhao $\mathrm{H}$ et al (2016) Gene-based pleiotropy across migraine with aura and migraine without aura patient groups. Cephalalgia Int $\mathbf{J}$ Headache 36:648-657. doi:10.1177/0333102415591497 